\title{
Coherence of the lattice polarization in large-polaron motion
}

\author{
E. N. Myasnikov, ${ }^{1}$ A. E. Myasnikova, ${ }^{2}$ and F. V. Kusmartsev ${ }^{3}$ \\ ${ }^{1}$ Rostov State Pedagogical University, 344082 Rostov-on-Don, Russia \\ ${ }^{2}$ Rostov State University, 344090 Rostov-on-Don, Russia \\ ${ }^{3}$ Loughborough University, Loughborough, Leicestershire LE11 3TU, United Kingdom
}

(Received 7 January 2005; revised manuscript received 26 September 2005; published 16 December 2005)

\begin{abstract}
Main problems of the large polaron theory are considered. We demonstrate that the problem of searching the ground stationary state of a system of coupled fields with translation-invariant Hamiltonian can have a solution of the form $f(\mathbf{r}-\mathbf{v} t), \mathbf{v} \rightarrow 0$, i.e., the solution with the spontaneously broken translational symmetry. Such a state can be a ground state of a large polaron in case of strong electron-phonon coupling when the spontaneous break of the translational symmetry results from the phonon vacuum deformation by the electric field of the charge carrier. The correctness of the classical representation of the polarization field in the theory of a strongly coupled large polaron is proved on the base of the theory of the quantum-coherent states of the phonon field. The use of this representation has enabled us to show that extremely high losses of the electron energy in dielectric parts of cold cathodes occurring when the carrier velocity is lower than the threshold for the single-phonon radiation are due to coherent phonon radiation by polarons like Cherenkov effect. It is this radiation that results in the predicted Thornber and Feynman dependence of the carrier steady-state velocity on the applied electric field strength. The coherent phonon radiation generated by polaron current can be detected in experiments on the neutrons scattering. The primary directions of the neutrons scattering depend on the polarons steady-state velocity and, hence, on the applied field strength. The coherent phonon radiation stemming from supersonic thermal motion of polarons causes a giant increase of the resistance in a corresponding temperature interval.
\end{abstract}

DOI: 10.1103/PhysRevB.72.224303

PACS number(s): 71.38. $-\mathrm{k}, 72.20 .-\mathrm{i}$

\section{INTRODUCTION}

The strong-coupling theory of a large polaron has had some unsolved problems from the very time of its creation. Two central problems are the following ones:

(1) Why do the theories with the classical and quantum consideration of the polarization field yield practically the same results for all experimentally observable polaron characteristics (e.g., binding energy, effective mass of the polaron)?

(2) Is the polaron state of a plane-wave type or should the polaron motion be described by a wave function of the type $\psi(\mathbf{r}-\mathbf{v} t)$ ?

In the present consideration we clarify them using a relatively young (in comparison with the polaron theory) quantum-coherent states theory and the conception of the spontaneous symmetry break. We demonstrate that the classical description of the phonon field in the large polaron is a reasonable approach in case of strong electron-phonon coupling (when the adiabatic condition is satisfied) since the phonon vacuum deformation by the field of the electron plays the main role in the polaron formation. This deformation breaks the system translational symmetry and after the polaron formation the system is not invariant with respect to the group of spatial and time translations. But it is invariant with respect to the subgroup of the combined spatial-time translations, and the polaron wave function has the form of $\psi(\mathbf{r}-\mathbf{v} t)$.

Then on the base of these two conclusions and as an experimental confirmation of them we consider Thornber and Feynman effect ${ }^{1}$ and give its interpretation. Thornber and
Feynman ${ }^{1}$ prove the possibility of motion of the polaron with extremely high losses when its velocity is considerably less than the threshold velocity for the single-phonon radiation. They have found ${ }^{1}$ that extremely high losses of the charge carrier energy in dielectric parts of the cold-cathode devices are caused by particular processes of the optical phonon scattering of polarons. Then this result obtained in Ref. 1 with the path-integral method was rederived with other methods. ${ }^{2-4}$ In the present work we demonstrate that the mentioned particular process of the optical phonon scattering of polarons is a coherent phonon radiation by a moving polaron that occurs when its velocity exceeds the minimum phase velocity of phonons. Similar processes have a fundamental importance not only in the polaron theory, they are well-known since the creation of Landau Bose-liquid theory ${ }^{5}$ and the theory of Cherenkov radiation. ${ }^{6,7}$ Thornber and Feynman stress that the radiation ${ }^{1}$ is so intensive that there is not a possibility to distinguish the acts of single-quantum radiation. The same is well known for the case of Cherenkov radiation which represents a reaction of the medium to the field of a moving carrier. ${ }^{7}$ A common base for these processes description is Landau Bose-liquid theory. ${ }^{5}$ It states that if there is relative motion of Bose vacuum with respect to a particle that can be an origin of the coordinate system then this motion generates a radiation when the velocity of the motion exceeds the minimum phase velocity of Bose particles.

A coordinate system cannot be related to a particle being in a state with a certain momentum but it can be related with a localized particle, for example, with a polaron. Here it is worth noting that Landau and Pekar have constructed their theory of the large polaron ${ }^{8}$ using the classical description of 
the polarization field breaking the translational symmetry of the medium. It is historically the first theory of interaction of two quantized fields (electron and phonon) resulting in a spontaneous break of their symmetry. In this theory the fact that the state of a resting polaron is degenerated with respect to the polaron center position does not mean a possibility of the transition to plane-wave states of the polaron, the classical field prevents this transition. In such a theory the degeneration with respect to the polaron location leads simply to a possibility of translation of the polaron center without external influence (by the inertia).

Below we shall demonstrate that transition to quantum consideration of the phonon field does not change this statement. To prove this one should take into account that the state of a quantized boson field of phonons can be changed in two ways. One of them is a deformation of the vacuum of a harmonic (i.e., a shift of the equilibrium value of its coordinate and momentum ${ }^{9}$ ) and the other one is a change of the number of phonons in the harmonic. The former is described by the quantum-coherent state theory. ${ }^{9-11}$ It, in particular, shows that quantum averages of physical values in a coherent state develop in time according to classical laws. ${ }^{9}$ If the energy of the vacuum deformation is much higher than that of phonons then the system can be considered in neglecting the latter ones. We shall demonstrate below that deformation of the phonon vacuum by the field of the electron plays the main role in formation of the strongly coupled large polaron. Therefore the state of the polarization field in such a polaron can be considered in neglecting quantum fluctuations of the polarization related with the presence of the certain number of quanta in the harmonics, i.e., classically, as it is done by Landau-Pekar theory.

The paper is organized in the following way. Section II recalls the properties of the coherent states important for the polaron theory. Section III considers the problem of the symmetry of two coupled fields system in a homogeneous space and time. Section IV represents the theory of strongly coupled large polaron in terms of the coherent states. Section $\mathrm{V}$ considers the motion of such a polaron. Section VI explores the coherent phonon radiation arising due to the motion of large polarons and demonstrates that losses due to this radiation coincide with ones obtained by Thornber and Feynman. Section VII considers the temperature behavior of the system resistance and, in particular, its colossal increase in a certain temperature interval due to the coherent phonon radiation by thermal polarons.

\section{COHERENT STATES AND THEIR PROPERTIES}

The theory of the coherent states has been developed in physics of lasers. ${ }^{9,10}$ This theory is created to analyze states of a harmonic oscillator or a system of harmonics as, for example, the photon field or the phonon field. Below we recall the properties of the coherent states that are important for the further consideration.

(1) Coherent states $\left|p_{\mathbf{k}}, q_{\mathbf{k}}\right\rangle$, where $p_{\mathbf{k}}$ and $q_{\mathbf{k}}$ are the average values of the momentum and coordinate, respectively, are eigenstates of Hamiltonian
$H_{\mathbf{k}}\left(p_{\mathbf{k}}, q_{\mathbf{k}}\right)=\frac{1}{2 M} P_{\mathbf{k}}^{2}+\frac{M \omega_{\mathbf{k}}^{2}}{2} Q_{\mathbf{k}}^{2}-P_{\mathbf{k}} p_{\mathbf{k}} / M-M \omega_{\mathbf{k}}^{2} Q_{\mathbf{k}} q_{\mathbf{k}}-\frac{\hbar \omega_{\mathbf{k}}}{2}$

corresponding to its minimum eigenvalue $E=-\left(p_{\mathbf{k}}^{2} / 2 M\right.$ $\left.+M \omega_{\mathbf{k}}^{2} q_{\mathbf{k}}^{2} / 2\right){ }^{9}$ Hamiltonian (1) is known as Hamiltonian of a shifted harmonic oscillator. In the present consideration the harmonic oscillator is supposed to be the $k$ th harmonic of the phonon field, $Q_{\mathbf{k}}$ is its normal coordinate, $P_{\mathbf{k}}$ is the operator of its momentum $\left(P_{\mathbf{k}}=-i \hbar^{-1} \partial / \partial Q_{\mathbf{k}}\right)$.

(2) Following Ref. 9, let us denote $|0\rangle$ the eigenfunction of Hamiltonian of the nonshifted oscillator

$$
H_{\mathbf{k}}(0,0)=\frac{P_{\mathbf{k}}^{2}}{2 M}+\frac{M \omega_{\mathbf{k}}^{2}}{2} Q_{\mathbf{k}}^{2}-\frac{\hbar \omega_{\mathbf{k}}}{2}
$$

corresponding to its ground state. The unitary operator that transforms the Hamiltonian of the nonshifted oscillator into one of the shifted oscillator (and the vector $|0\rangle$ into $\left|p_{\mathbf{k}}, q_{\mathbf{k}}\right\rangle$ ) has the form ${ }^{9,11}$

$$
\begin{gathered}
U\left(p_{\mathbf{k}}, q_{\mathbf{k}}\right)=\exp \left[i \hbar^{-1}\left(p_{\mathbf{k}} Q_{\mathbf{k}}-q_{\mathbf{k}} P_{\mathbf{k}}\right)\right]=\exp \left(b_{\mathbf{k}}^{+} d_{\mathbf{k}}-b_{\mathbf{k}} d_{\mathbf{k}}^{*}\right), \\
\mathbf{d}_{\mathbf{k}}=\frac{q_{\mathbf{k}}+i p_{\mathbf{k}}}{\sqrt{2 \hbar / M \omega_{\mathbf{k}}}}=\left|\mathbf{d}_{\mathbf{k}}\right| \exp \left(i \varphi_{\mathbf{k}}\right),
\end{gathered}
$$

where $b_{\mathbf{k}}^{+}, b_{\mathbf{k}}$ are the operators of creation and annihilation of quanta in the $k$ th harmonic, respectively; $\varphi_{\mathbf{k}}$ in (3) is, obviously, the harmonic phase. It should be noted that in a state with the certain number $N_{\mathbf{k}}$ of quanta in a harmonic, the phase of the harmonic is completely uncertain due to the uncertainty relation $\Delta \varphi_{\mathbf{k}} \Delta N_{\mathbf{k}}>1 .^{12}$

Operator (2) transforms also the operators $b_{\mathbf{k}}^{+}, b_{\mathbf{k}}$, into new ones $b_{\mathbf{k}}^{+\prime}=b_{\mathbf{k}}^{+}-d_{\mathbf{k}}, b_{\mathbf{k}}^{\prime}=b_{\mathbf{k}}-d_{\mathbf{k}}$ in such a way that in a state $\left|p_{\mathbf{k}}, q_{\mathbf{k}}\right\rangle$ (Ref. 11),

$$
\left\langle b_{\mathbf{k}}^{\prime}\right\rangle=0, \quad\left\langle b_{\mathbf{k}}\right\rangle=d_{\mathbf{k}} .
$$

Thus, this transformation distinguishes a classical field $d_{k}$ in each harmonic and introduces it into the consideration. In the case of the electromagnetic field it can be considered as Bose condensate of photons. The classical field arises only at high intensity of the interaction that can induce the appearance of the shifts $d_{k}$, in the field harmonics. As the theory of lasers shows this field breaks the system translational symmetry and represents the order parameter for the system transition into the regime of generation of the laser radiation as the pumping level increases. ${ }^{13}$ Haken $^{13}$ has demonstrated the analogy between the transition of a laser into the generation regime and a phase transition in a solid. The system behavior in the generation regime (including the near-threshold regime) at different pumping levels is described well without taking into account quantum fluctuations. ${ }^{10}$ The phonon field of the crystal is similar to the electromagnetic field as both of them are boson fields and both of them have observable physical characteristics with corresponding operators which are linear combinations of the operators of creation and annihilation of quanta. Hence, results obtained in the theory of 
lasers can be applied to a phonon field strongly coupled with the electron.

\section{SYMMETRY OF A CLOSED SYSTEM OF COUPLED FIELDS IN A HOMOGENEOUS SPACE}

It is ordinarily considered that states of a closed system of coupled fields in a homogeneous space obey the symmetry of a certain subgroup of the group of translations. However, this statement does not take into account the fact that such a system is invariant with respect to translations in time. Taking this fact into account changes the situation completely as the whole group of the spatial and time translations has a subgroup of combined translations. If the wave function of such a field in Cartesian coordinate system has the form

$$
\exp [i(\mathbf{k r}-\omega t)]=\exp \left\{i\left[k_{x} x+k_{y} y+k_{z} z-\omega(\mathbf{k}) t\right]\right\}
$$

then it automatically takes into account both translational symmetries of the system in space and in time. It is easy to note that this function is invariant with respect to the group of spatial-time translations with the element

$$
\exp 2 \pi\left(\frac{n_{1}}{k_{x}} \frac{\partial}{\partial x}+\frac{n_{2}}{k_{y}} \frac{\partial}{\partial y}+\frac{n_{3}}{k_{z}} \frac{\partial}{\partial z}-\frac{n_{4}}{\omega} \frac{\partial}{\partial t}\right),
$$

where $n_{i}, i=1-4$, are arbitrary integer (including zero) parameters, distinguishing different elements of the group (5). The above wave function is a product of the function of spatial coordinates and the function of time. Each of the multipliers is invariant with respect to a subgroup of spatial or time translations. Therefore, one can consider that in such a system the dependence of the wave function on spatial coordinates has the form of $\exp (i \mathbf{k r})$.

But this choice is not unique. The group of spatial-time translations has a subgroup of combined translations with the element

$$
\exp t_{0}\left(v_{x} \frac{\partial}{\partial x}+v_{y} \frac{\partial}{\partial y}+v_{z} \frac{\partial}{\partial z}-\frac{\partial}{\partial t}\right)=\exp \left[t_{0}(\mathbf{v} \nabla-\partial / \partial \mathbf{t})\right],
$$

where $t_{0}$ is an arbitrary value which distinguishes different elements of the group (6). The set of parameters $\left(v_{x}, v_{y}, v_{z}\right)$ distinguishes one such subgroup from another. Obviously, any function $f(\mathbf{r}-\mathbf{v} t)$ is invariant with respect to the subgroup (6). Indeed, each element of the group (6) transforms $f(\mathbf{r}-\mathbf{v} t)$ into the form $f\left[\mathbf{r}+\mathbf{v} t_{0}-\mathbf{v}\left(t+t_{0}\right)\right]=f(\mathbf{r}-\mathbf{v} t)$. Thus, in the systems homogeneous in the space and time the states of the form $f(\mathbf{r}-\mathbf{v} t)$ can exist as well, and these states are not invariant with respect to the subgroup of spatial translations. In such states the parameter $\mathbf{v}$, not the momentum, as it takes place in the plane-wave states (i.e., in the states of the type of $\left.e^{i(\mathbf{k r}-\omega \mathbf{t})}\right)$, has a certain value. Therefore one can imagine an energy band of such a system formed by states with different values of the velocity $\mathbf{v}$. So, we have proved that the problem of searching the ground stationary state of a system of coupled fields with translation-invariant Hamiltonian can have a solution of the form $f(\mathbf{r}-\mathbf{v} t), \mathbf{v} \rightarrow 0$.

This conclusion does not contradict the group theory. Indeed, the states of the system can be "irreducible," i.e., de- scribed by the wave functions spanning one irreducible representation of the group of symmetry of the system Hamiltonian. But (in more complicated cases, e.g., as it is shown in the next section, at strong electron-phonon interaction) they can also be "reducible," described by a wave function spanning the linear combination of the functions related to different irreducible representations of the same group. The form of the linear combination is determined by the system Hamiltonian. In the case under consideration a delocalized state is described by the wave function proportional to $e^{i(\mathbf{k r}-\omega \mathbf{t})}$, whereas the wave function of the localized state can be expanded in terms of the plane waves with different wave vectors essentially incommensurate with each other. As a consequence, such a solution does not possess the translational invariance.

Thus, translational invariance of Hamiltonian (or its commutation with the momentum operator) does not automatically mean that the wave function of the ground state will be an eigenfunction of the momentum operator. In some works on the polaron theory one can meet the opposite incorrect statement. For example, the work ${ }^{14}$ on the strong-coupling theory of the polaron uses (in its Appendix) the statement that the exact polaron eigenstates simultaneously diagonalize both energy and momentum. The same idea is contained in the supposition of Ref. 15 that a density matrix of the charge carrier in a large polaron does not depend on the coordinate of the electron. The author of Ref. 16 looks for a polaron ground state only among the eigenstates of the operator of the polaron momentum (i.e., among the states of the form $\left.e^{i \mathbf{k r}}\right)$. This supposition does not allow the authors of Refs. 15 and 16 to find a transition from the plane-wave states of the polaron to the states with spontaneously broken translational symmetry as the electron-phonon coupling increases.

In fact a commutation of two operators means simply that they have a common system of eigenfunctions but it does not warrant that one of these eigenfunctions will correspond to the ground state. Depending on the character and on the strength of the fields interaction the state characterized by the functions of the type $f(\mathbf{r}-\mathbf{v} t)$, i.e., the states with the spontaneously broken symmetry with respect to spatial translations, can be more energetically profitable. (This occurs in case of the strongly coupled large polaron as it is shown in the next section. In such a case the system of eigenfunctions of the Hamiltonian turns out to be overcrowded like the system of eigenfunctions of shifted harmonic oscillator or the one of the electromagnetic field. ${ }^{9}$ ) Therefore, searching stationary states of motion of homogeneous closed systems, it is not correct to prefer a priori the states with the wave functions of the type $e^{i(\mathbf{k r}-\omega \mathrm{t})}$. One should necessarily study the solutions that are invariant with respect to the subgroup of the combined translations and then choose the true ground state by comparing the energies of solutions of the both types. Such a procedure was carried out, for example, by Holstein in his well-known works: ${ }^{17}$ he obtains both delocalized and localized solutions and found that the lowest in the energy solution is the localized one.

The question whether the spatially homogeneous system can appear in a spatially inhomogeneous state is like, for example, the well-known question about the break of the inversion symmetry of the atomic nucleus (the break of the 
spatial parity conservation law) in processes caused by weak interaction. This phenomenon, as it was shown by Landau, results from the fact that the nucleus is invariant also with respect to the operation of time inversion, and the transition with the broken parity is invariant with respect to the combined inversion. It is also worth noting here that the distribution of the type $f(\mathbf{r}-\mathbf{v} t)$ is characteristic for the motion of classical systems in a homogeneous spacetime.

\section{COHERENCE OF THE POLARIZATION FIELD IN A STRONGLY COUPLED LARGE POLARON}

As it was shown by Landau and Pekar, ${ }^{8}$ a classical field of the lattice deformation that breaks the system translational symmetry can arise due to strong electron-phonon interaction resulting in the large polaron formation. The analogous classical field of shifts of the equilibrium positions of the lattice normal modes is introduced in a fundamental work on the small polaron theory by Holstein. ${ }^{17} \mathrm{He}$ constructs a molecular-crystal model to study small polaron properties. In Ref. 17 it is also demonstrated that if one replaces the shortrange interaction term in the Hamiltonian of this model by long-range interaction then the model becomes equivalent to the continuum-polarization model of the large-polaron theory by Landau and Pekar until a supposition about the bare electron bandwidth is made. If the carrier bandwidth is much larger than the polaron binding energy then an increase of the electron kinetic energy with decreasing the polaron radius does not allow the polaron to shrink to a size of a crystal cell. In the opposite case we deal with the small polaron.

Holstein has also pointed out that to study such processes as a polaron motion dispersion of the lattice vibration frequencies should be taken into account. To treat the resulting Hamiltonian the vibrational coordinates are expressed in terms of the normal-mode coordinates of the crystal. Then Holstein Hamiltonian (except for the carrier kinetic energy) has the form ${ }^{17}$

$$
H_{L}+H_{i}=\sum_{\mathbf{k}}\left(\frac{P_{\mathbf{k}}^{2}}{2 M}+\frac{1}{2} M \omega_{\mathbf{k}}^{2} Q_{\mathbf{k}}^{2}-M \omega_{\mathbf{k}}^{2} Q_{\mathbf{k}} q_{\mathbf{k}}^{(n)}\right)
$$

$q_{\mathbf{k}}^{(n)}$ in Ref. 17 denotes a shift of the equilibrium position in the $k$ th harmonic when the carrier is situated on the $n$th site; the difference between the cases of short-range and longrange interaction is only in the form of $q_{\mathbf{k}}^{(n)}$.

It is easy to see, that (7) differs from Hamiltonian (1) of a shifted harmonic oscillator with $p_{k}=0$ only in the reference point of energy by the value $\hbar \omega_{\mathbf{k}} / 2$. Therefore, it is convenient to express its eigenstates in terms of the quantumcoherent states that are eigenstates of a shifted harmonic oscillator. As it will be shown below Landau-Pekar Hamiltonian and Holstein Hamiltonian can be obtained from Frohlich Hamiltonian if only the coherent part of the polarization field is retained in the latter. (It should be noted that this coherent part differs from zero only in the case of strong electron-phonon coupling.) Frohlich Hamiltonian reads (except the electron kinetic energy operator)

$$
\begin{aligned}
H_{L}+H_{i}= & \sum_{\mathbf{k}} \hbar \omega_{\mathbf{k}} b_{\mathbf{k}}^{+} b_{\mathbf{k}}-\sum_{\mathbf{k}} \frac{e}{|\mathbf{k}|} \sqrt{\frac{2 \pi \hbar \omega_{\mathbf{k}}}{V \widetilde{\varepsilon}}}\left[b_{\mathbf{k}} \exp (i \mathbf{k r} \mathbf{r})\right. \\
& \left.+b_{\mathbf{k}}^{+} \exp (-i \mathbf{k r})\right],
\end{aligned}
$$

where $\mathbf{r}$ is the electron coordinate, $V$ is a crystal volume, $\widetilde{\varepsilon}$ is an effective dielectric permittivity of the medium, $\widetilde{\varepsilon}^{-1}=\varepsilon_{\infty}^{-1}$ $-\varepsilon_{0}^{-1}, \varepsilon_{0}$ and $\varepsilon_{\infty}$ are static and optical dielectric constants, respectively.

In the case of strong electron-phonon interaction average values of the operators of creation and/or annihilation of phonons in (8) are different from zero according to the property of the shift operator [item (2) of Sec. II]. To analyze states of the phonon field in the polaron it is useful to distinguish the shift of the harmonics equilibrium positions in a clear form. To do this let us transit to operators $b_{k}^{\prime}, b_{k}^{+\prime}$ related with the initial ones as follows: $b_{\mathbf{k}}=b_{\mathbf{k}}^{\prime}+d_{\mathbf{k}}, b_{\mathbf{k}}^{+}=b_{\mathbf{k}}^{+\prime}$ $+d_{\mathbf{k}}^{*}$, where $\left\langle b_{\mathbf{k}}\right\rangle=\left\langle d_{\mathbf{k}}\right\rangle,\left\langle b_{\mathbf{k}}^{+}\right\rangle=\left\langle d_{\mathbf{k}}^{*}\right\rangle,\left\langle b_{\mathbf{k}}^{\prime}\right\rangle=0,\left\langle b_{\mathbf{k}}^{+}\right\rangle=0$. After this transformation (8) has the form (below we shall omit the strokes)

$$
\begin{aligned}
H_{L}+H_{i}= & \sum_{\mathbf{k}} \hbar \omega_{\mathbf{k}}\left(b_{\mathbf{k}}^{+} b_{\mathbf{k}}+b_{\mathbf{k}}^{+} d_{\mathbf{k}}+d_{\mathbf{k}}^{*} b_{\mathbf{k}}+d_{\mathbf{k}}^{*} d_{\mathbf{k}}\right) \\
& -\sum_{\mathbf{k}} \frac{e}{|\mathbf{k}|} \sqrt{\frac{2 \pi \hbar \omega_{\mathbf{k}}}{V \widetilde{\varepsilon}}}\left[b_{\mathbf{k}} \exp (i \mathbf{k r})+b_{\mathbf{k}}^{+} \exp (-i \mathbf{k r})\right. \\
& \left.+\left|\mathbf{d}_{\mathbf{k}}\right| 2 \cos \left(\mathbf{k r}+\varphi_{\mathbf{k}}\right)\right] .
\end{aligned}
$$

Polarization corresponding to Hamiltonian (8) has the form

$$
\mathbf{P}(\mathbf{n})=i \sum_{\mathbf{k}} \sqrt{\frac{\hbar}{2 M V \omega_{\mathbf{k}}}} \frac{\mathbf{k}}{|\mathbf{k}|} \exp (i \mathbf{k n})\left(b_{\mathbf{k}}+b_{\mathbf{k}}^{+}\right),
$$

where $\mathbf{n}$ is the number of the crystal cell. After the transition to operators of creation and/or annihilation of phonons with zero average values the polarization operator has the form

$$
\begin{aligned}
\mathbf{P}(\mathbf{n}) & =i \sum_{\mathbf{k}} \sqrt{\frac{\hbar}{2 M V \omega_{\mathbf{k}}}} \frac{\mathbf{k}}{|\mathbf{k}|} \exp (i \mathbf{k n})\left(b_{\mathbf{k}}+b_{\mathbf{k}}^{+}+d_{\mathbf{k}}+d_{\mathbf{k}}^{*}\right) \\
& =i \sum_{\mathbf{k}} \sqrt{\frac{\hbar}{2 M V \omega_{\mathbf{k}}}} \frac{\mathbf{k}}{|\mathbf{k}|} \exp (i \mathbf{k n})\left(b_{\mathbf{k}}+b_{\mathbf{k}}^{+}+\left|\mathbf{d}_{\mathbf{k}}\right| 2 \cos \varphi_{\mathbf{k}}\right) .
\end{aligned}
$$

As it is ordinarily made in the theory of the strongly coupled large polaron ${ }^{8}$ we shall seek the ground state wave function in the form of the product of electron and phonon wave functions:

$$
|s\rangle=\psi_{0}(\mathbf{r}-\mathbf{R}) \exp \left(\sum_{\mathbf{k} \neq 0}\left(d_{\mathbf{k}} b_{\mathbf{k}}^{+}-d_{\mathbf{k}}^{*} b_{\mathbf{k}}\right)\right)|0\rangle,
$$

where $|0\rangle$ is the vector of the ground state of phonon subsystem in absence of the phonon vacuum deformation, $\mathbf{R}$ is a vector of the real space, and $\psi_{0}(\mathbf{r})$ is a normalized electronic wave function corresponding to the polaron ground state. Let us represent the shift parameter in the following form: $d_{\mathbf{k}}$ $=\left|d_{\mathbf{k}}\right| e^{i \varphi_{\mathbf{k}}}$. Obviously, one should find the shift parameters $\left|d_{\mathbf{k}}\right|, \varphi_{\mathbf{k}}$ and a localization parameter in $\psi_{0}(\mathbf{r})$ by minimization of the functional 


$$
\begin{aligned}
\langle s|H| s\rangle= & \left\langle-\frac{\hbar^{2}}{2 m^{*}} \nabla_{\mathbf{r}}^{2}\right\rangle+\sum_{\mathbf{k} \neq 0}\left[\hbar \omega _ { \mathbf { k } } \left(d_{\mathbf{k}}^{*} d_{\mathbf{k}}+\left\langle s\left|b_{\mathbf{k}}^{+} b_{\mathbf{k}}\right| s\right\rangle\right.\right. \\
& \left.\left.-\frac{e}{|\mathbf{k}|} \sqrt{\frac{2 \pi \hbar \omega_{\mathbf{k}}}{V \widetilde{\varepsilon}}} d_{\mathbf{k}} e^{i \mathbf{k r}}+d_{\mathbf{k}}^{*} e^{-i \mathbf{k r}}\right)\right] .
\end{aligned}
$$

Here $\left\langle s\left|b_{\mathbf{k}}^{+} b_{\mathbf{k}}\right| s\right\rangle=0$ as it is the average number of phonons in the $k$ th harmonic in the ground state.

The solution of the equation $\partial\langle s|H| s\rangle / \partial\left|d_{\mathbf{k}}\right|=0$ has the form

$$
\left|\mathbf{d}_{\mathbf{k}}\right|=\frac{e}{|\mathbf{k}|} \sqrt{\frac{2 \pi}{V \widetilde{\varepsilon} \hbar \omega_{\mathbf{k}}}} \cos \left(\mathbf{k} \mathbf{R}+\varphi_{\mathbf{k}}\right) \eta(\mathbf{k}),
$$

where $\mathbf{R}$ is the radius vector of the polaron center, $\eta(\mathbf{k})$ is the Fourier transform of the function $|\psi(\mathbf{r}-\mathbf{R})|^{2}$. Substituting (13) into (12) one can obtain

$$
\langle s|H| s\rangle=\left\langle-\frac{\hbar^{2}}{2 m^{*}} \nabla_{\mathbf{r}}^{2}\right\rangle-\sum_{\mathbf{k} \neq 0} \frac{e^{2}}{\mathbf{k}^{2}} \frac{2 \pi}{V \widetilde{\varepsilon}} \cos ^{2}\left(\mathbf{k} \mathbf{R}+\varphi_{\mathbf{k}}\right) \eta^{2}(\mathbf{k}) .
$$

Minimum of (14) corresponds to

$$
\varphi_{\mathbf{k}}=-\mathbf{k} \mathbf{R}+2 \pi C(\mathbf{k R}),
$$

where $C(\mathbf{k} \mathbf{R})$ is such an integer that $\varphi_{\mathbf{k}} \in[-\pi, \pi]$. It is apparent that the phase (15) is expressed through the parameters $\mathbf{k}$ and $\mathbf{R}$ having definite values. Hence, in the state $|s\rangle$ corresponding to the minimum of $\langle s|H| s\rangle$ the polarization field is quantum coherent. It breaks translational symmetry of the system.

Finally, choosing the test wave function in the form

$$
\psi(\mathbf{r}-\mathbf{R})=[\mathbf{1}+\alpha(\mathbf{r}-\mathbf{R})] \exp [-\alpha(\mathbf{r}-\mathbf{R})]
$$

one can find that for the value $\alpha$ found by Pekar ${ }^{8}$ the functional (14) achieves its extremum and yields the well-known value for the binding energy of the large polaron. Since, as the calculation shows, the localized state of the system of interacting electron and phonon fields is more energetically profitable in the case of strong electron-phonon interaction (in case of the adiabatic condition being satisfied), it is the system ground state.

Average value of the polarization in a state (13) reads

$$
\mathbf{P}(\mathbf{n})=i \sum_{\mathbf{k}} \sqrt{\frac{\pi}{M \widetilde{\varepsilon}}} \frac{2 e \mathbf{k}}{|\mathbf{k}|^{2} V \omega_{\mathbf{k}}} \exp (i \mathbf{k n}) \cos (\mathbf{k R}) \eta(\mathbf{k}) .
$$

As it is seen from (16) all the harmonics of the polarization field in the large polaron have certain phases, i.e., this field is coherent, or classical. A distinguished point of the classical polarization field with the radius-vector $\mathbf{R}$ indicates the center of the electron localization. Let us stress that in the coherent state of the polarization field occurring in the large polaron a nonzero average value of the polarization is observable in each cell of the crystal and changes regularly as the polaron moves (i.e., as $\mathbf{R}$ changes with time). Analogously, in the coherent state of the electromagnetic field in a laser a regular change of the average electric field strength

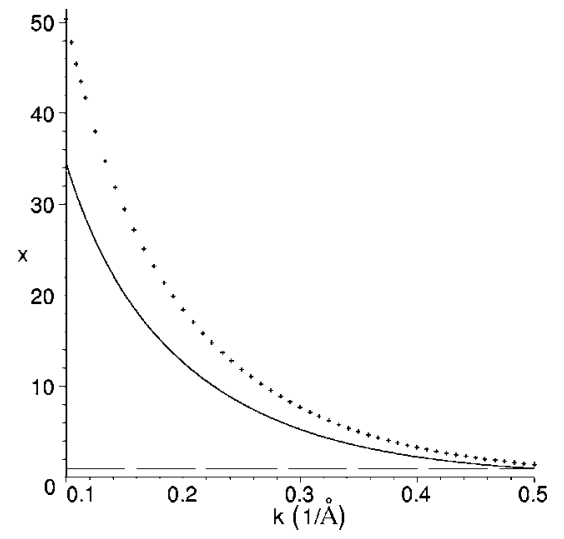

FIG. 1. Ratio of the shift of the equilibrium position of a phonon field harmonic in the large polaron and the mean-square declination from the average value in this harmonic as a function of the harmonic wave vector. Dotted and solid lines correspond to the temperature $0.01 \mathrm{eV}$ and $0.03 \mathrm{eV}$, respectively. Dashed line shows $x$ $=1$ level.

occurs, that is impossible for the field of a photon with a certain momentum.

Now we can test the correctness of the approximation made in Ref. 8. Let us compare the obtained value $q_{k}$ of the shift of the equilibrium position in each harmonic due to strong electron-phonon interaction with the mean-square displacement $\xi_{k}$ from the average value in this harmonic. For a polaron in rest (3) and (13) yield

$$
q_{\mathbf{k}}=\left|d_{\mathbf{k}}\right| \sqrt{2 \hbar / M \omega_{\mathbf{k}}}=\frac{e}{|\mathbf{k}|} \sqrt{\frac{2 \pi}{V \widetilde{\varepsilon} \hbar \omega_{\mathbf{k}}}} \sqrt{\frac{2 \hbar}{M \omega_{\mathbf{k}}}} \eta(\mathbf{k}) .
$$

As is known from quantum mechanics

$$
\xi_{\mathbf{k}}=\sqrt{\left(\frac{1}{e^{\hbar \omega_{\mathbf{k}} / k T}-1}+\frac{1}{2}\right) \frac{\hbar}{M N \omega_{\mathbf{k}}}}
$$

where $N$ is the number of cells in the crystal. It is easy to see by the calculation that for any medium where the adiabatic condition $\left(E_{\mathrm{pol}} \gg \hbar \omega,{ }^{8}\right.$ where $E_{\mathrm{pol}}$ is the polaron binding energy) is satisfied, the condition $q_{k} \gg \xi_{k}(T)$ is satisfied for all the harmonics participating in the polaron formation.

For example, Fig. 1 demonstrates the value of a ratio $x$ $=q_{k} / \xi_{k}$ as a function of the wave vector $\mathbf{k}$ for two values of the system temperature and typical values of the inverse effective dielectric permittivity $1 / \widetilde{\varepsilon}=0.3$, free carrier effective mass $m^{*}=m_{e}$, phonon energy $\hbar \omega=0.05 \mathrm{eV}$, and lattice constant $a=4 \AA$. As it is seen from Fig. 1, $x \gg 1$ for the polaron harmonics with the wave vectors $k<0.32 \AA^{-1}$. They are just the harmonics contributing to the polaron as one can see from the value of the polaron radius in this medium. The same is correct for any other medium parameters satisfying the adiabatic condition.

Thus, neglecting the incoherent part of the phonon field, i.e., using classical description of the polarization field in the large polaron, is a reasonable approximation in case of strong electron-phonon interaction. It is interesting to note that 
within the limit of zero temperature the condition $q_{k}$ $\gg \xi_{k}(T)$ being squared and summarized for all the harmonics yields the condition $8 E_{\mathrm{pol}} / \hbar \omega \gg 1$ [we take into account (13) and (14) and the fact that $E_{\mathrm{pol}}=-\left(H_{L}+H_{i}\right) / 2$ (Ref. 8)], which is much weaker than the adiabatic condition.

\section{MOTION OF A STRONGLY COUPLED LARGE POLARON}

Let us now discuss how a strongly coupled large polaron can move. It cannot exist in a plane-wave state since in such a state the square of the wave function modulus has the same value in all cells of the crystal. In such a case the quantum average of the electric field of the charge carrier is zero in all points of the space. Such a field cannot generate coherent polarization. If the carrier state is localized then the quantum average of the electron electric field is different from zero and this field can generate coherent polarization.

As it is shown in Sec. III the conclusion about impossibility of a plane-wave state for the strongly coupled large polaron does not contradict the symmetry of the system. Moreover, in a complete accordance with Goldstone theorem disappearance of the translational invariance results in an appearance of a large (infinite in the case of continuous translational invariance) number of degenerated states of the resting polaron. They are transformed one into another by the disappeared symmetry element and, therefore, differ by the value of the polaron center coordinate $\mathbf{R}$. The polaron motion breaks this degeneration since the polaron motion expresses itself in a change of the vector $\mathbf{R}$ in (13) and (16) with time. Motion of the strongly coupled large polaron is possible if the phonon vacuum deformation (i.e., the coherent part of the polarization field) formed due to carrier interaction with phonons can accompany the carrier translational motion. Let us consider under what conditions this occurs.

According to the theory of quantum coherent states if shifting term(s) in Hamiltonian (7) are time dependent then the state $\left|p_{\mathbf{k}}(t), q_{\mathbf{k}}(t)\right\rangle$ is an eigenvector of the time-dependent Hamiltonian of shifted harmonic oscillator in each moment of time, and the corresponding eigenvalue is $E_{\mathbf{k}}=E_{\mathbf{k}}(t)$ $=-\left[p_{\mathbf{k}}^{2}(t)+q_{\mathbf{k}}^{2}(t)\right] / 2,{ }^{9}$ i.e., quantum averages of physical values in a coherent state develop in time as solutions of classical equations of motion (as it is characteristic for the motion of wave packets $\left.{ }^{18}\right)$. These equations are well known and for the density of the polarization charge $\rho(\mathbf{r}, t)=-\nabla \mathbf{P}(\mathbf{r}, t)$ generated by a moving carrier with the charge density $e|\psi[\mathbf{r}-\mathbf{R}(t)]|^{2}$ the equation of motion has the form: ${ }^{19}$

$$
\left(\partial^{2} / \partial t^{2}+\Omega^{2}-u^{2} \nabla_{\mathbf{r}}^{2}\right) \rho(\mathbf{r}, t)=e \widetilde{\varepsilon}^{-1} \Omega^{2}|\psi(\mathbf{r}-\mathbf{R}(t))|^{2},
$$

provided the phonon dispersion reads $\omega^{2}(\mathbf{k})=\Omega^{2}+u^{2} \mathbf{k}^{2}$. For the case of $\mathbf{R}(t)=\mathbf{v} t$ a Green function $G(\mathbf{r}, t)$ for Eq. (17) [i.e., its solution when the function $|\psi[\mathbf{r}-\mathbf{R}(t)]|^{2}$ is replaced by $\delta[\mathbf{r}-\mathbf{R}(t)]]$ can be obtained analytically. Obviously, $G(\mathbf{r}, t)$ represents the polarization charge generated by a moving point free charge. Since we consider the case when the adiabatic condition is satisfied it is quite natural that polarization reacts to the average electron charge distribution that is expressed, in particular, in the following expression:

$$
\rho(\mathbf{r}, t)=-e \widetilde{\varepsilon}^{-1} \Omega^{2} \int G\left(\mathbf{r}-\mathbf{r}^{\prime}\right) \psi^{2}\left(\mathbf{r}^{\prime}, t\right) d \mathbf{r} .
$$

It turns out that the form of the Green function is completely different in two regions of the polaron velocity modulus $v$. When $v$ is lower than the minimum phase (or maximum group) velocity $u$ of phonons, $G(\mathbf{r}, t)$ is localized in the vicinity of "generating" free charge. In a cylindrical coordinate system with $z$-axis lying along $\mathbf{v}$ it has a form: ${ }^{19}$

$$
\begin{gathered}
G(\mathbf{r}, t)_{v \leqslant u}=\exp \left(-\Omega l_{1} / u\right) / 4 \pi u^{2} \beta_{1} l_{1}, \\
l_{1}=\sqrt{(z-v t)^{2} / \beta_{1}^{2}+r^{2}}, \quad \beta_{1}=\sqrt{1-v^{2} / u^{2}} .
\end{gathered}
$$

In such a case interaction of free and polarization charges results in a polaron formation. If $v>u$ the polarization charge is not localized but oscillates at infinity in a cone $z$ $-v t<0 ; r<|z-v t| / \beta_{2}$ behind the free charge, indicating appearance of a coherent phonon radiation,

$$
\begin{gathered}
G(\mathbf{r}, t)_{v>u}=\cos \left(\Omega l_{2} / u\right) / 2 \pi u^{2} \beta_{2} l_{2}, \\
l_{2}=\sqrt{(z-v t)^{2} / \beta_{2}^{2}-r^{2}}, \quad \beta_{2}=\sqrt{v^{2} / u^{2}-1} .
\end{gathered}
$$

Thus, a coherent polarization caused by electron-phonon coupling can accompany the motion of a point charge particle moving with the velocity $v<u$. Hence, according to (18) the strongly coupled large polaron can move with the velocities lower than the minimum phase velocity of phonons participating in its formation. The energy of moving polaron depends on its velocity. In the effective mass approximation this dependence can be represented in a form $E_{\mathrm{pol}}(v)=E_{\mathrm{pol}}(0)+M_{\mathrm{pol}}^{*} v^{2} / 2$, where the polaron effective mass $M_{\mathrm{pol}}$ depends on its velocity, and this dependence is quasi relativistic as the velocity approaches the minimum phase velocity of phonons ${ }^{20}$ If the average velocity of the carrier is higher than the minimum phase velocity of phonons the coherent polarization cannot accompany its motion and the polaron state breaks. Thus, we can conclude that the energy band of the strongly coupled large polaron is formed by polaron states of the form $f(\mathbf{r}-\mathbf{v} t)$ with different values of the polaron velocity from the interval $0 \leqslant|\mathbf{v}| \leqslant u$. If, however, there is another factor of localization of a carrier moving with the average velocity $v>u$ (for example, its strong interaction with another phonon branch characterized by a higher minimum phase velocity) then we can get into the region of the polaron velocities where a coherent phonon radiation is possible. This is realized in the next section.

Some supporters of the idea of the plane-wave states of the strongly coupled polaron consider (following the known case of the hydrogen atom) that the solution of the type $f(\mathbf{r}$ $-\mathbf{v} t$ ) describes the carrier motion along the relative coordinate $\mathbf{r}=\mathbf{r}_{1}-\mathbf{r}_{2}$ of the electron and phonon wave packets, whereas the plane-wave behavior takes place along the coordinate of their center of masses. However, the spatial coordinate in the phonon field harmonic is not a dynamical variable that is why one cannot construct dynamical variables describing relative motion of these subsystems and the motion of their center of mass from the electron spatial coordinate and the coordinate in the harmonic of the phonon field. 
Therefore the idea that in case of the polaron the electron localization occurs along the coordinate of the relative motion and the plane-wave state takes place along the centerof-masses coordinate, contradicts the laws of the quantum mechanics. The electron localization in the polaron occurs in a space of its own coordinates, and the wave function of the carrier in the polaron has the form $f(\mathbf{r}-\mathbf{v} t)$. In a plane-wave state the carrier is delocalized (its wave function $\propto \exp (-i \mathrm{pr} / \hbar))$ and is unable to produce a classical polarization of the medium.

Thus, in case of strong electron-phonon interaction the large polaron is an autolocalized state of the charge carrier with the spontaneous break of the crystal translational symmetry. After the spontaneous break of the system translational symmetry it remains symmetric with respect to the combined spatial-time translations. The autolocalized carrier state can move in a crystal with a velocity $v$ lower than the minimum phase velocity $u$ of phonons since the coherent polarization can accompany the localized carrier motion while its velocity does not exceed this limit. The energy of moving polaron depends on its velocity and changes inside some band when $v$ changes into the interval $0 \leqslant|\mathbf{v}| \leqslant u$.

Such a movable autolocalized state of a carrier is an alternative to a plane-wave state. Indeed, as it was pointed out above the autolocalized state of the carrier can exist only in case of strong electron-phonon interaction, as it was pointed out by Landau. ${ }^{8}$ The condition of the autolocalized carrier state existence is the value of the Frohlich constant of the electron-phonon interaction much higher than the unity. Everything given above allows stating that the region of strong electron-phonon coupling is the region where the autolocalized state of the charge carrier is its ground state. In the region of weak coupling a plane-wave state of the carrier is its ground state. The transition from one type of states to the other one has not been found in works Refs. 15 and 16 since their authors searched eigenstates of the polaron Hamiltonian only among the plane-wave states. The present paper shows that the eigenstate with the spontaneously broken translational symmetry corresponds to more deep minimum of the system energy. Only a theory taking into account both coherent and incoherent parts of the phonon field can describe this transition correctly.

\section{COHERENT PHONON RADIATION CAUSED BY "SUPERSONIC" MOTION OF STRONGLY COUPLED LARGE POLARONS}

Coherence of the polarization field of the large polaron is also confirmed by the effect predicted in essence by Landau Bose-liquid theory and its application to the superfluidity phenomenon. It turns out that in a complete correspondence with this theory there exists a critical velocity $u$ of the relative motion of localized carrier and classical field of the lattice polarization that can also be called the Bose condensate of phonons. If the velocity $v$ of the localized carrier exceeds this critical one then intensive phonon radiation arises in the Bose condensate. The condition $v>u$ appearing in the superfluidity and/or superconductivity theory, in the theory of Cherenkov effect and in the present consideration directly points out that we deal not with a single-phonon radiation (the necessary condition $m^{*} v^{2} / 2>h \omega$ ) but with an excitation of the vacuum.

This effect in the large polaron theory was considered for the first time in the work of Thornber and Feynman. ${ }^{1}$ It is aimed to explain experimental data on the giant rate of the carrier energy loss in oxide covering of the cold-cathode devices. We demonstrate below that losses to the radiation calculated in the work of Thornber and Feynman are caused by coherent radiation of the polarization waves.

As it was shown in the preceding section, in a medium with one optical phonon branch there is not any factor of the carrier autolocalization if its average velocity $v>u$. Therefore a polaron can move in such a medium only with the velocity lower than the minimum phase velocity $u$ of phonons. To study the coherent phonon radiation by moving polaron and losses to it the model should contain some other factor of localization of the carrier with the velocity exceeding the minimum phase velocity of phonons. (In principle, theoretical studies can leave this factor not concretized concretizing only the resulting state of the charge carrier.) Obviously, the most probable such factor is strong interaction of the carrier with another phonon branch provided this branch has a higher minimum phase velocity of phonons. Thus we arrive at a model of medium with a two-component polarization. ${ }^{19}$

In this model of a medium two branches of polar phonons are supposed to exist. One of them has higher frequency $\omega$ and phonon velocity $u$ and strongly interacts with the charge carrier. It provides formation of the large polaron able to move with the velocity $v<u$. The other branch is characterized by smaller values of the frequency and phonon velocity $\left(\omega_{1}<\omega, u_{1}<u\right)$ and its coupling with the charge carrier (characterized by effective dielectric constant $\widetilde{\varepsilon}^{\prime}$ ) can be arbitrary. In such a medium two types of polarons can exist. Following notations in Ref. 19 let us call them double polaron (DP) and single polaron (SP). Both phonon branches contribute to DP whereas only the branch with the higher minimum phase velocity contributes to SP formation. DP can move with the velocity $v$ from the interval $0 \leqslant v \leqslant u_{1}$. SP exists in the velocity region $u_{1}<v<u$ and its motion with such velocities generates a coherent radiation of phonons of the branch $\omega_{1}$.

It is easy to calculate the losses due to the radiation per unit length. To do it let us consider stationary motion of the polaron in an electric field with the field strength $\mathbf{E}$. The projection of the braking force on the direction of motion reads

$$
F_{z}=\int G_{1}\left(\mathbf{r}-\mathbf{r}_{1}\right) \psi^{2}\left(\mathbf{r}_{1}\right) \psi^{2}\left(\mathbf{r}_{2}\right) \frac{z-z_{2}}{\left|\mathbf{r}-\mathbf{r}_{2}\right|^{3}} d \mathbf{r} d \mathbf{r}_{1} d \mathbf{r}_{2},
$$

where $G_{1}$ is a Green function determined by (20), but with $u$, $\omega$ replaced by $u_{1}, \omega_{1}$. One can readily calculate $F_{z}$ using Fourier transforms; the Fourier transform of the Green function is 


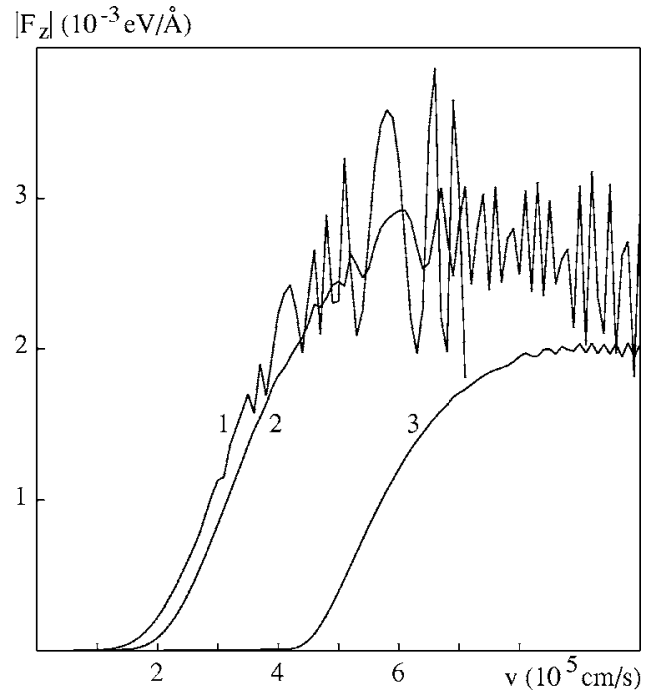

FIG. 2. $F_{z}(v)$ curves in a medium with $\widetilde{\varepsilon}^{-1}=0.3, \widetilde{\varepsilon}^{-1}=0.03$, $m^{*}=m_{e}, \omega_{1}=10^{13} \mathrm{~s}^{-1}, \eta=0.01 \omega_{1}$, and $u=10^{6} \mathrm{~cm} / \mathrm{s}$. Curves 1,2 , and 3 correspond to the case of $u_{1}=2.5 \times 10^{4}, 10^{5}$, and 4 $\times 10^{5} \mathrm{~cm} / \mathrm{s}$, respectively. Curve 1 is cut at $7 \times 10^{5} \mathrm{~cm} / \mathrm{s}$ because of strong oscillations.

$$
G_{1}(k)=\frac{1}{k_{z}^{2} v^{2}-\left(\omega_{1}^{2}+u_{1}^{2} k^{2}+u_{1}^{2} k_{z}^{2}\right)+i \gamma k_{z} v}
$$

where $\gamma$ is a small parameter that characterizes the damping. Pekar wave function ${ }^{8}$ was used as a carrier wave function in the polaron.

Figure 2 depicts the dependence of $\left|F_{z}\right|$ on the polaron velocity $v$ [or $E(v)$ dependence where $v$ is the modulus of the steady-state carrier velocity and $E$ is the field strength necessary to maintain it] for three values of $u_{1}$. As (19) and (20) demonstrate the condition of the radiation appearance is $v>u_{1}$. However, the gradual increase of the function $E(v)$ from near zero up to the maximum takes place at higher values of $v$ when the interference of the phonon waves radiated by different parts of the polaron becomes constructive. It occurs when the radiation wavelength exceeds more or less the polaron radius $R_{\mathrm{SP}}$. As is seen from (20) the wavelength of the radiation caused by an element of the polaron volume depends on the polaron velocity, $\lambda=2 \pi\left(v^{2}-u_{1}^{2}\right)^{1 / 2} / \omega_{1}$. Therefore the value of $v$ corresponding to sharp increase of the losses is $v^{2}>u_{1}^{2}+\left(\omega_{1} R_{\mathrm{SP}} / 2 \pi\right)^{2}$. When $u_{1}^{2}$ in the right-hand part is small in comparison with the second summand the ascending parts of the $\left|F_{z}\right|$ curves coincide. The braking force increases with the increasing polaron velocity while the polarization charge wave is forming owing to the interference. Then $\left|F_{z}\right|$ decreases with the continuing increase in velocity due to the first maximum of the polarization charge wave moving away from the polaron. The interference of the waves radiated by different parts of the polaron causes oscillations of the function $F_{z}(v)$. The braking force depends also on $\widetilde{\varepsilon}^{\prime}$ and $\widetilde{\varepsilon}$ values and on the polaron radius $R_{\mathrm{SP}}$. For typical values of the medium parameters used to calculate Fig. 2 the braking force can be as large as $2 \times 10^{-3} \mathrm{eV} / \AA$. Hence, for the SP stationary motion the electric field strength of the

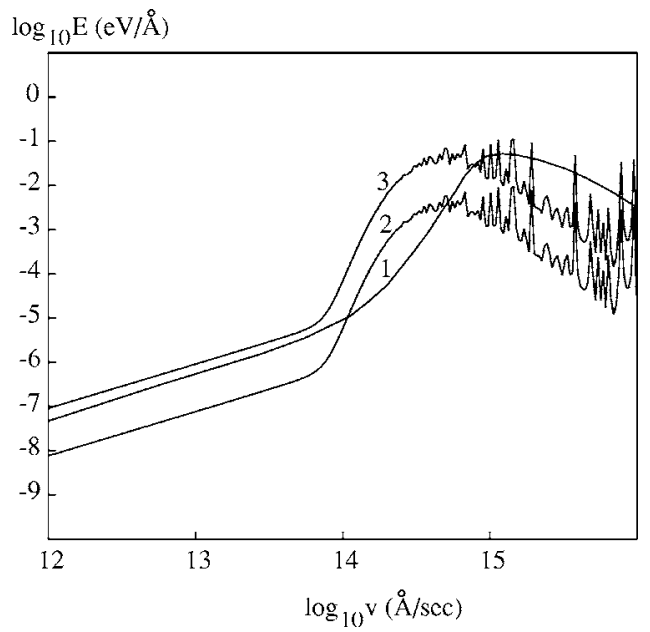

FIG. 3. $E(v)$ curves in a logarithmic scale. Curve 1 is calculated in Ref. 1 for the case of $\alpha=5, m^{*}=m_{e}$ (that corresponds to $c$ $=0.357), T=0.1 \hbar \omega / k_{B}\left(\beta=10\right.$, where $\left.\beta=E_{\mathrm{ph}} / k_{B} T\right), \hbar \omega=0.07 \mathrm{eV}$. Curve 2 is obtained by (21) for the same medium parameters as curve 1 (including $c=0.357$ ) and $c_{1}=0.03, u_{1}=10^{5} \mathrm{~cm} / \mathrm{s}, u \gg u_{1}$. Curve 3 is calculated by (21) for the same medium parameters as curve 2 except for $c_{1}=0.357$.

order of $10^{5} \mathrm{~V} / \mathrm{cm}$ is necessary. Thus, coherent phonon radiation can be observed in dielectric parts of the coldcathode devices in such fields.

After a transformation on the sample boundary the phonon radiation can be fixed as a coherent electromagnetic radiation with a frequency of the order of phonon frequencies. Therefore the properties of the phonon radiation are here of interest too. The Green function (20) allows to find the angle $\theta$ between the direction of the polaron motion and the radiation direction, $\theta=\arccos \left(u_{1} / v\right)$, analogously to the Cherenkov radiation case. The frequency $\omega_{\text {rad }}$ of the phonon radiation depends on the polaron velocity, $\omega_{\mathrm{rad}}=\omega_{1} / \sqrt{1-u_{1}{ }^{2} / v^{2}}$, and, consequently, on the applied field, as determined by Fig. 2 . It should be noted that only the ascending part of the $\left|F_{z}(v)\right|$ dependence corresponds to a stable equilibrium, and it is this part that should be used to determine the field strength necessary to achieve a certain SP velocity. To avoid the influence of the thermal motion very low temperatures are required.

The coherent phonon radiation generated by polaron current can be detected in experiments on the neutrons scattering. A simple estimate shows that if the neutrons temperature is of the order of $1 \mathrm{~K}$ then a change of their initial momentums resulting from scattering on the coherent phonon radiation will be perceptible. Primary direction of the scattering (the direction of the radiation wave vector) makes an angle $\theta$ with the direction of the polaron current. As it has been shown above this direction depends on the polaron steadystate velocity and, hence, on the applied field strength.

Figure 3 enables to compare the results of Thornber and Feynman ${ }^{1}$ and ones of the present calculations. Curve 1 in Fig. 3 depicts the $E(v)$ dependence calculated in Ref. 1 for the case of $\alpha=5$, phonon energy $E_{\mathrm{ph}}=0.07 \mathrm{eV}$, temperature $T=0.1 E_{\mathrm{ph}} / k_{B}$ (in Ref. 1 this curve is denoted as $\beta=10$, where $\left.\beta=E_{\mathrm{ph}} / k_{B} T\right), m^{*}=m_{e}$, where $m^{*}$ is the electron band 
mass, and $T$ is the temperature. Curve 2 in Fig. 3 demonstrates the $E(v)$ function calculated by (21) for the same values of $\alpha$ (it corresponds to $\widetilde{\varepsilon}^{-1}=0.357$ ), $m^{*}$ and $E_{\mathrm{ph}}$ $=\hbar \omega_{1}$ and following other medium parameters: $\widetilde{\varepsilon}^{\prime-1}=0.03$, $u_{1}=10^{5} \mathrm{~cm} / \mathrm{s}, \eta=0.001 \Omega_{1}, u \gg u_{1}$. As was shown above (see Fig. 2 discussion), at such values of the $\omega_{1} / u_{1}$ ratio $E(v)$ curve does not differ from one in the case of $u_{1}=0$ used in Ref. 1 The value of $\eta$ influences only the linear (in the logarithmic scale) part of the curve.

Figure 3 clearly demonstrates the similar character of the $E(v)$ curves calculated by (21) and obtained by Thornber and Feynman $^{1}$ [interference oscillations on the $E(v)$ curve in Ref. 1 may be masked by presence of thermal phonons taken into account there]. However, there are some quantitative differences between them caused by a difference in the models. We use a medium model with the two-component polarization whereas a single dispersionless branch of longitudinal optic phonons was assumed to occur in Ref. 1. Moreover, in our model only a small part of the whole polarization (associated with the phonon branch $\omega_{1}$ and therefore proportional to $\widetilde{\varepsilon}^{\prime-1}=0.03$ ) is responsible for the phonon radiation while the largest part (associated with the branch $\omega$ and, thus, proportional to $\left.\widetilde{\varepsilon}^{-1}=0.357\right)$ provides the autolocalization of the carrier. In the Thornber and Feynman model the whole polarization (proportional to $\widetilde{\varepsilon}^{-1}=0.357$ ) is radiated. Therefore to reproduce the value of the electric field strength in the curve's 1 maximum in the calculation by (21) it is sufficient to take $\widetilde{\varepsilon}^{\prime-1}=0.357$ with all other values of the system parameters remaining unchanged. The result is demonstrated by curve 3 in Fig. 3 .

The value of the polaron velocity corresponding to the maximum of the $E(v)$ curve is determined by the size of the carrier localization region. [It is also clearly seen from results of Ref. 1 demonstrating a shift of the threshold region on the $E(v)$ curves to lower electron velocity as the electron-phonon coupling increases, and, accordingly, the polaron radius decreases.] In our model a charge carrier having nonzero average velocity remains in the polaron state. As it is clearly demonstrated by (19) and (20), in the Thornber and Feynman model the polarization apparently cannot provide localization of a moving carrier because the model contains a single dispersionless (i.e., $u=0$ ) phonon branch. However, characteristic features of the radiation obtained in their work (its high intensity at the carrier velocities below the threshold for the single-phonon radiation but above the value of the minimum phase velocity of phonons, as it is seen from Fig. 2, and such a high rate of losses that it is impossible to distinguish separate acts of a single-phonon radiation, which is emphasized in Ref. 1) unambiguously indicate to the coherent character of the radiation. The same features are characteristic for Cherenkov radiation. ${ }^{6,7}$ As it is demonstrated above only the motion of a localized charge carrier can generate coherent radiation. Hence, the charge carrier in the Thornber-Feynman model is localized. In all likelihood, it is a wave packet. Therefore, it is natural that its size is different from the polaron one that manifests itself in a shift of the position of the maximum of curve 1 with respect to the maxima of curves 2 and 3. Thus, the results of the work of Thornber and Feynman confirm the idea about large polaron at strong coupling as about autolocalized state of the charge carrier.

It should be noted that the form of $E(v)$ curves obtained by Thornber and Feynman ${ }^{1}$ with the path-integral method was then reproduced using Heisenberg equations of motion. ${ }^{2,3}$ The low-velocity portion of the $E(v)$ dependence of a similar character has also been obtained analytically ${ }^{4}$ by using the Bogolyubov-Tyablikov method. Although the authors of Ref. 4 used a model with a single dispersionless phonon branch, that, according to (20), makes the motion of the strong-coupling large polaron impossible, they nevertheless assumed the polaron to exist at its velocities $v>0$ (it is equivalent to the presence of another phonon branch strongly interacting with the charge carrier and having a large dispersion). In spite of this drawback of the model the authors of Ref. 4 came to the important conclusion that the dependence $E(v)$ obtained by them was associated with a new mechanism of the carrier energy loss.

The results obtained in this section are of importance in two respects.

(1) The coherent nature of the phonon radiation in the Thornber-Feynman effect is proved which opens a possibility of its experimental study and, possibly, of its practical application.

(2) The statement about possibility of classical description of the coherent states in the polaron theory in case of strong coupling of the carriers and the lattice deformation in neglecting the role of the quantized field is confirmed once more.

\section{BRAKING OF POLARONS BY THE COHERENT PHONON RADIATION AND COLOSSAL INCREASE OF THE RESISTANCE}

As it was demonstrated in Sec. V, a velocity of the strongly coupled large polaron is limited by a minimum phase velocity of phonons participating in its formation. This limitation on the polaron velocity causes thermal destruction of polarons at temperatures much lower than the polaron binding energy. ${ }^{19,21}$ Indeed, the average temperature of the polaron destruction (in a model with single-component polarization) is approximated in Refs. 21 and 22 as $T_{c}$ $=0.278 E_{\mathrm{pol}}\left(m^{*} u / \widetilde{\varepsilon} p_{0}\right)^{0.176}$, where $p_{0}$ is the maximum momentum of a carrier in the polaron in a polaron model with constant distribution of the probability density to find the carrier provided the mean-square deviation of the carrier from its average position is the same as in the polaron described by the Pekar wave function; $p_{0}$ is much larger than the maximum momentum $m^{*} u$ of the carrier translational motion. ${ }^{23}$ At temperatures higher than $T_{c}$ charge carriers are mainly in free (plane-wave) states where their effective mass is considerably smaller than one in the polaron state. Therefore in a medium where a charge carrier interacts (strongly) with a single branch of optic phonons the system resistance will demonstrate "activation" behavior in the corresponding region of temperature near $T_{c}{ }^{22}$

In a medium with two-component polarization like that described in Sec. VI one can predict more complicated behavior of the system resistance. Indeed, in such a system two types of polarons (DP and SP) can exist. According to results 
of Ref. 21 DPs predominate at low temperatures then, as the temperature increases, DPs lose one polarization "coat" and SPs become a dominant carrier state and, with the further increase of temperature, all the carriers turn out to be in free (plane-wave) states. Transition from SP states to free carrier states as the temperature increases will be accompanied by activational behavior of the system resistance. But considering the transition from DP into SP states apart from the effect of change of the carrier effective mass one should take into account that the motion of SP is accompanied by coherent phonon radiation.

In absence of a strong electric field the braking force caused by the radiation determines the SP drag acceleration $\left|a_{\mathrm{SP}}\right|=\left|F_{z}(v)\right| / M_{\mathrm{SP}}(v)$, where $M_{\mathrm{SP}}$, i.e., the effective inertial mass of the SP, is characterized by a quasirelativistic dependence on the polaron velocity. ${ }^{20}$ According to the theory of electrical conductivity the average ratio of the polaron velocity to its acceleration is the polaron relaxation time. Hence, if in some temperature region the SPs play a significant role in the conductivity one can predict in this temperature interval a large decrease in the carrier mobility and the corresponding large increase in the resistance like one in materials that exhibit colossal magnetoresistance. ${ }^{24}$ The temperature interval where SPs are predominant is determined by the binding energies of the DP and SP and by the values of $u_{1}$ and $u .^{21,22}$

Let us calculate the temperature dependence of the resistance in the considered above system with the twocomponent polarization. The SP relaxation time is

$$
\tau_{\mathrm{SP}}=\overline{v_{\mathrm{SP}}} / \overline{a_{\mathrm{SP}}(v)}=\overline{p_{\mathrm{SP}}} / \overline{\left|F_{z}(v)\right|} .
$$

To determine the average SP momentum and the average braking force projection we make use of the distribution function in a system of the coexisting DPs, SPs and free carriers. $^{21}$ The inverse value of the relaxation time calculated by (22) should be added to the inverse time of the SP relaxation in ordinary scattering processes. ${ }^{25}$ Relaxation time of DPs and free carriers was calculated by the well-known formulas $^{25,26}$ as it was done in Ref. 22.

Figure 4 demonstrates the temperature dependence, calculated in this manner, of the resistance in a system with $\widetilde{\varepsilon}^{\prime-1}$ $=0.03, \widetilde{\varepsilon}^{-1}=0.3, m^{*}=m_{e}$ for two sets of the minimum phase velocities of phonons.

A large increase in the resistance results from the coherent phonon radiation by SPs. The magnitude of the resistance increase produced by the phonon radiation due to the thermal motion of SPs is determined by $\widetilde{\varepsilon}$ and $\widetilde{\varepsilon}^{\prime}$ and values whereas its temperature position depends mainly on the values of $u_{1}$ and $u$. Subsequent decrease of the resistance with the rising temperature is due to gradual transition of carriers from the SP into free carrier states characterized by a considerably smaller carrier effective mass.

\section{CONCLUSION}

In summary, the coherent state theory applied to a description of the strongly coupled large polarons has shown that such polarons break the system translational symmetry by their field of the lattice deformation. The autolocalized carrier state can move in a crystal with a velocity $v$ lower

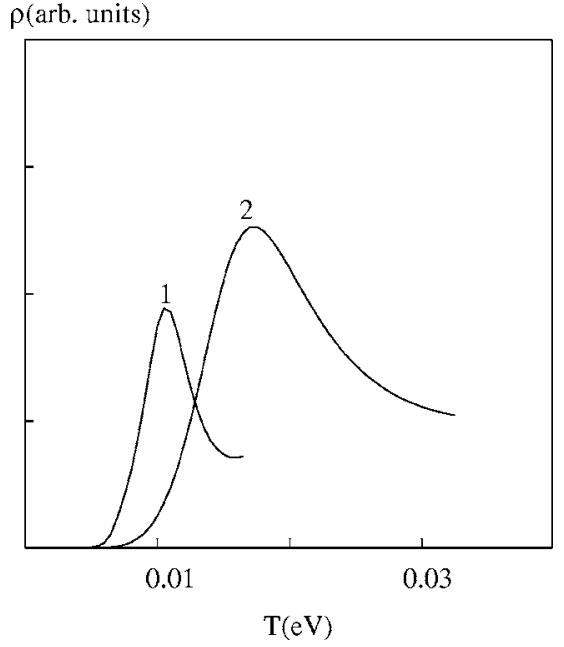

FIG. 4. Temperature dependence of the resistance in a system with two-component polarization. Curves 1 and 2 correspond to the case of $u_{1}=10^{5} \mathrm{~cm} / \mathrm{s}, u=5 \times 10^{5} \mathrm{~cm} / \mathrm{s}$ and $u_{1}=10^{6} \mathrm{~cm} / \mathrm{s}, u=5$ $\times 10^{6} \mathrm{~cm} / \mathrm{s}$, respectively. The scale on the ordinate axis for curve 2 is 10 times larger.

than the minimum phase velocity $u$ of phonons since the coherent polarization can accompany the localized carrier motion while its velocity does not exceed this limit. The energy of moving polaron depends on its velocity and changes inside some band when $v$ changes into the interval $0 \leqslant|\mathbf{v}| \leqslant u$.

If the polaron velocity is higher than the minimum phase velocity of phonons then in a medium with two-component polarization a mechanism of the polaron braking by the coherent phonon radiation occurs. It is this mechanism that causes the extremely high losses of the carrier energy in dielectric parts of the cold-cathode devices studied by Thornber and Feynman ${ }^{1}$ with the path integral method. The use of the classical consideration of the polarization field has enabled us to prove that the radiation obtained in their work is the coherent phonon radiation of the medium under the influence of the field of moving localized carrier like Cherenkov effect. The resulting braking of the carrier is so powerful that for a compensation of losses of the polaron kinetic energy the electric field with the field strength of the order of $10^{5} \mathrm{~V} / \mathrm{cm}$ is necessary.

One can expect that experimental detection of the coherent phonon radiation generated by polaron current in experiments on scattering of slow neutrons is possible. Change of the neutrons momentums will be perceptible if the neutrons temperature is about $1 \mathrm{~K}$. Primary direction of the neutrons scattering on the coherent phonon radiation determined by external normal to the radiation front will demonstrate dependence on the applied electric field. Experimental study of the coherent phonon radiation is of interest, since its application in construction of the electromagnetic radiation sources with the frequencies of the order of $10^{13} \mathrm{~s}^{-1}$ may be possible.

In the region of temperatures where the large polarons thermal velocities exceed the lowest minimum phase velocity of phonons the systems of large polarons in a medium with two-component polarization demonstrate giant increase 
of the resistance. At higher temperatures an "activational" temperature dependence of the conductivity occurs in such systems due to the polarons destruction when their thermal velocities become higher than the minimum phase velocity of phonons interacting with the carrier.

\section{ACKNOWLEDGMENT}

The authors are grateful to F. M. Peeters for calling our attention to relevant works, and S. B. Rochal for helpful discussions.
${ }^{1}$ K. K. Thornber and R. P. Feynman, Phys. Rev. B 1, 4099 (1970).

${ }^{2}$ F. M. Peeters and J. T. Devreese, Phys. Rev. B 23, 1936 (1981).

${ }^{3}$ F. M. Peeters and J. T. Devreese, Solid State Phys. 38, 81 (1984).

${ }^{4}$ V. V. Bryksin, V. V. Onuchin, A. V. Prokaznikov, and G. Yu. Yashin, Sov. Phys. Solid State 30, 261 (1988).

${ }^{5}$ L. D. Landau, Zh. Eksp. Teor. Fiz. 11, 592 (1941); J. Phys. (Moscow) 11, 91 (1947).

${ }^{6}$ I. E. Tamm and I. M. Frank, Dokl. Akad. Nauk SSSR 14, 107 (1937).

${ }^{7}$ V. L. Ginsburg, Zh. Eksp. Teor. Fiz. 10, 589 (1940).

${ }^{8}$ L. D. Landau, Phys. Z. Sowjetunion 3, 664 (1933); L. D. Landau and S. I. Pekar, Zh. Eksp. Teor. Fiz. 18, 419 (1948); S. I. Pekar, Untersuchungen uber die Electronentheorie der Kristalle (Akademie-Verlag, Berlin, 1954).

${ }^{9}$ J. R. Clauder and E. C. G. Sudarshan, Fundamentals of Quantum Optics (Benjamin, New York, 1968).

${ }^{10}$ M. Lax, Fluctuation and Coherence Phenomena in Classical and Quantum Physics (Gordon and Breach, New York, 1968).

${ }^{11}$ H. Haken, Quantenfeldtheorie des Festkorpers (Teubner, Stutgart, 1973).

${ }^{12} \mathrm{~W}$. Heitler, The Quantum Theory of Radiation (Clarendon, Oxford, 1954).
${ }^{13}$ H. Haken, Festkoerperprobleme 10, 351 (1970).

${ }^{14}$ G. R. Allcock, in Polarons and Excitons, edited by C. G. Kuper and G. D. Whitfield (Oliver and Boyd, Edinburg, 1963), pp. 45-70.

${ }^{15}$ B. Gerlach and H. Lowen, Phys. Rev. B 35, 4291 (1987).

${ }^{16}$ H. Lowen, Phys. Rev. B 37, 8661 (1988).

${ }^{17}$ T. Holstein, Ann. Phys. 8, 325 (1959); 8, 343 (1959).

${ }^{18}$ P. A. M. Dirac, The Principles of Quantum Mechanics (Clarendon, Oxford, 1958).

${ }^{19}$ A. E. Myasnikova, Phys. Rev. B 52, 10457 (1995).

${ }^{20}$ A. E. Myasnikova and E. N. Myasnikov, Phys. Rev. B 56, 5316 (1997); JETP 88, 101 (1999).

${ }^{21}$ E. N. Myasnikov and A. E. Myasnikova, JETP 89, 746 (1999).

${ }^{22}$ A. E. Myasnikova, Phys. Lett. A 291, 439 (2001).

${ }^{23}$ E. N. Myasnikov and A. E. Myasnikova, Phys. Lett. A 286, 210 (2001).

${ }^{24}$ W. E. Pickett and D. J. Singh, Phys. Rev. B 53, 1146 (1996).

${ }^{25}$ J. Appel, "Polarons," in Solid State Physics: Advances in Research and Applications (Academic, New York, 1968) Vol. 21.

${ }^{26}$ A. I. Anselm, Introduction into the Theory of Semiconductors (Nauka, Moscow, 1978). 